\title{
APPLICATION OF ELECTRODYNAMIC TREATMENT WITH AMPLITUDE-FREQUENCY CONTROL OF CURRENT PULSES FOR LIFE EXTENSION OF AIRCRAFT STRUCTURES OF LIGHT ALLOYS*
}

\author{
L.M. LOBANOV ${ }^{1}$, N.A. PASHCHIN ${ }^{1}$, O.L. MIKHODUJ ${ }^{1}$, A.V. CHERKASHIN ${ }^{1}$, \\ A.V. ZARUTSKY ${ }^{2}$ and I.P. KONDRATENKO ${ }^{3}$ \\ ${ }^{1}$ E.O. Paton Electric Welding Institute, NASU \\ 11 Kazimir Malevich Str., 03680, Kiev, Ukraine. E-mail: office@paton.kiev.ua \\ ${ }^{2}$ N.E. Zhukovsky National Aerospace University «KhAI» \\ 17 Chkalov Str., Kharkov, Ukraine \\ ${ }^{3}$ Institute of Elecrodynamics, NASU \\ 56 Pobeda Ave., 03057, Kiev, Ukraine
}

\begin{abstract}
The pulsed electric current (PEC) generator of a new generation with the controlled amplitude-frequency characteristics of PEC for electrodynamic treatment (EDT) of thin-sheet metal structures was designed and manufactured. The use of a new class of equipment allows a significant extension of capabilities of electrodynamic effects due to the controlled synchronization of the EDT dynamic and electric pulse components. The effect of EDT on reducing the level of residual welding stresses in magnesium alloy ML10 joints was investigated. As the investigated specimens the fragments of the shell of the intermediate body of the aircraft engine D-36 containing repair welds were used. It was found that EDT allows reducing the level of residual welding stresses from 120 to -40 MPa, providing their transition from tensile to compressive ones. It is shown that the local plastic deformation applying EDT of specimens of an aircraft wing stringer of aluminum alloy D16 in the area of technological holes 1.6-2.5 times increases the resistance to a delayed fracture. 9 Ref., 2 Tables, 14 Figures.
\end{abstract}

Ke yword s : electrodynamic treatment, electric current pulse, aircraft structures, residual stresses, repair welding, body of aircraft engine, stringer, technological hole

At the repair of technological and service defects in the aircraft structures of magnesium alloys the nonconsumable electrode welding in argon (TIG) is used [1]. One of the causes for deterioration of service characteristics of products is residual stresses in repair joints reducing the life of vehicles. The presence of residual stresses causes a need in postweld heat treatment of structures in the large-sized electric furnaces, which requires significant energy costs and increases the cost of repair operations. In this case heat treatment does not completely eliminate the probability of fatigue cracks in the repair welds. This fact facilitates the search and development of alternative methods for reduction of level of residual stresses in welded joints, one of which is the electrodynamic treatment (EDT) $[2,3]$, based on the simultaneous effect of current pulse and dynamic pressure of current-conducting electrode on product or weld. The application of EDT reduces the level of residual stresses in repair welds, which in many cases may be an alternative to heat treatment. It allows reducing the cost of repair technologies at restoration of aircraft structural elements.

At the same time, in the modern technologies of aircraft industry the relevant problem is hardening of technological holes in the glider load-carrying elements of aluminum alloys in order to increase their resistance to a delayed fracture [4]. The modern mechanical methods of hardening the holes, based on the surface plastic deformation, have several disadvantages, which can be eliminated using electrodynamic effects.

The aim of the present work is the investigation of EDT effect on control of the level of residual welding and technological stresses in the elements of aircraft structures of light alloys.

The equipment for EDT of structural materials and welded joints is mainly based on the pulse current

\footnotetext{
*According to the materials of the work performed within the frames of the target integrated program of the NAS of Ukraine «Problems of Life and Safe Operation of Structures, Constructions and Machines» (2013-2015).
} 

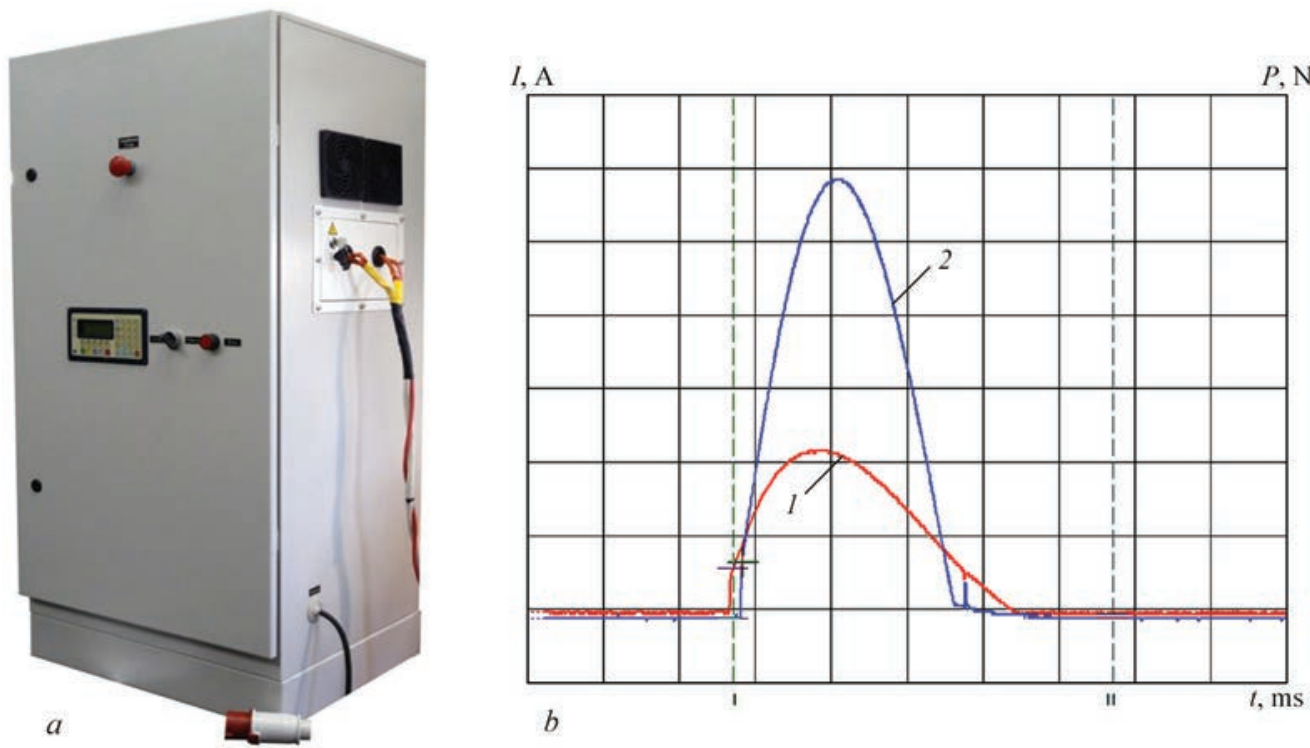

Figure 1. Pulsed current source «Iskra-4» for EDT of structural materials: $a$ - appearance; $b$ - oscillograms of current pulses of dynamic pressure 1 and direct current passing through the treated metal 2

source and electrode device providing current passing through the treatment zone and simultaneous controlled force effect on the treated surface. The treatment of welded joints of light alloys was carried out using pulsed current source of the second generation «Iskra-4» designed and manufactured at the Institute of Electrodynamics of the NAS of Ukraine.

The current pulse generator «Iskra-4», the appearance of which is shown in Figure 1, $a$, includes two independent channels, each of which includes a rectifier, charging inverter, capacitor and controlled discharge circuits.

The first channel of the pulse generator generates a current pulse 1 (Figure $1, b$ ), which is intended to create dynamic effects on the treatment zone and is supplied to the inductance coil in the electrode device
(ED), which is an operating tool for EDT (Figure 2). The second channel of the pulse generator forms a current pulse 2 correlated as to its duration with the duration of dynamic pressure (current pulse 1) to the treatment zone. The pulse current of the second channel is supplied directly to the ED electrode and transferred to the treated workpiece. The electric circuit of the second channel of the generator is closed through the contact cable, which is attached at the product.

As to its design, ED (Figure 2, $a$ ) includes electrode 1 , manufactured of refractory conductive material fixed in the yoke 3 using fixing devices 2 and 20. The yoke is connected to a disc of a non-ferromagnetic material 16, which supported against the frame 6 with the inductance coil 14 located in it with the builtin plane inducer 15 . The coil is fixed on the frame
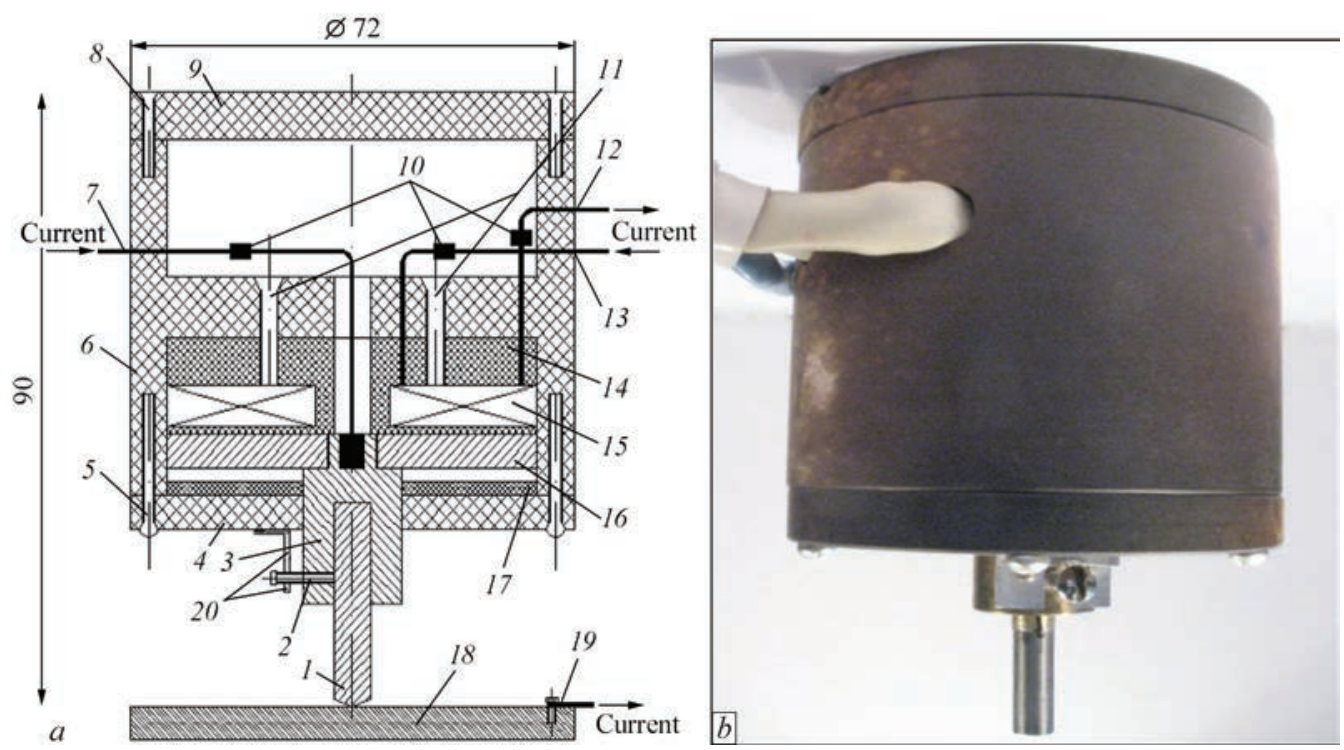

Figure 2. Electrode device for EDT with independent channels of current pulses: $a$ - design scheme (for designations see the text); $b$ - appearance 


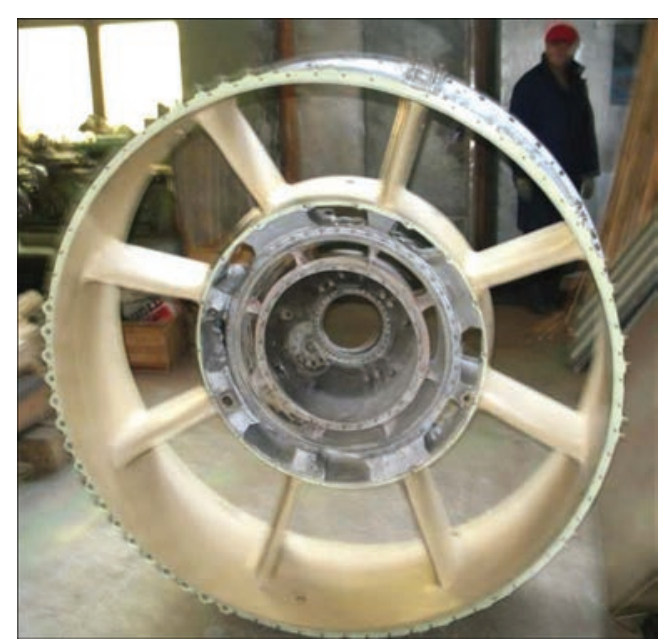

Figure 3. Appearance of intermediate body of aircraft engine of heat-resistant magnesium alloy ML10

with screws 11 . The bottom wall of the frame 4 , fixed by screws 5 , performs a role of non-magnetic backing under the disc and the damper 17. The upper (protective) cover 9 of the frame is fixed in the ED body with the screws 8 . The supply of the current pulse 2 (see Figure $1, b$ ) from the power source is carried out by the wire 7 . The short circuit of the discharge circuit providing the supply of the current pulse 2 to the treated metal 18 is carried out by the terminal 19 . The current pulse 1 (see Figure $1, b$ ) passes through the coil through the wires 12 and 13 . The supply wires 7, 12, 13 of ED are connected to the external circuits of the contact terminals 10. The appearance of ED is presented in Figure 2, $b$.

The operation of ED is carried out as follows. When a pulse current is passing through the coil the magnetic field is excited initiating eddy currents in the disc. The interaction of the induced currents with the magnetic field, which excited them, leads to generation of electrodynamic force. At the same time, in the first phase of effect, the electrodynamic force presses the electrode to the treated material, and in the second one the direction of force effect is changed to the opposite one, i.e. the electrode is repelled from the treated material. Obviously, that as to its duration the effect of the pulsed current on the treatment zone should not exceed the duration of clamping force (see Figure 1, $b$ ).

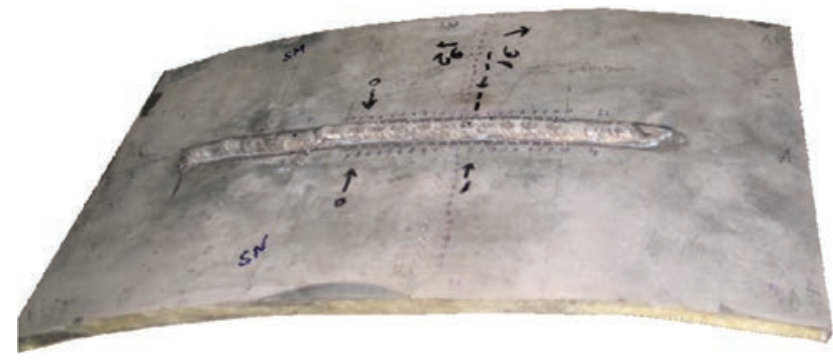

Figure 4. Specimen of repair welded joint of alloy ML10
Thus, the design of the pulsed current source is subjected to the requirements of the correlated pulse duration and exclusion of electrospark phenomena in the treatment zone. The control of channels in pulse generator «Iskra-4» is realized in three microcontrollers and programmable logics, which allow regulating current pulse parameters (amplitude), delay of one pulse with respect to the other, visualizing the pulse parameters on the light display. The pulse duration on both channels is determined by the parameters of electric circuit of discharge contours and can be changed discretely by adding/reducing the inductance contours. The additional inductances are installed on the outer side of the generator body, for which the contact groups on the side surface of the generator are provided.

In general, the basic technical characteristics of two-channel source of pulsed currents are as follows:

- power supply - three-phase mains of $380 \mathrm{~V}$ with a zero wire;

- galvanic input-output isolation — transformer of $2.5 \mathrm{kV}$;

- total installed capacity - $7 \mathrm{kVA}$;

- number of channels - 2;

- amplitude of current pulses in each channel up to $4.5 \mathrm{kA}(0-4.5 \mathrm{kA})$;

- current pulse duration in channel $1-680 \mu \mathrm{s}$;

- current pulse duration in channel $2-550 \mu \mathrm{s}$;

- phase shift of current pulses in the channels $10-100 \mu \mathrm{s}$;

- pulse repetition interval $-0.8-1.0 \mathrm{~s}$;

- operation mode - manual/automatic.

The investigations of EDT effect on regulation of residual stresses in welded joints of magnesium alloy were carried out. For this purpose, the specimens were used being the fragments of outer cylindrical shell of the intermediate body of the aircraft engine D-36 of $1.5 \mathrm{~m}$ diameter $[2,3]$ manufactured of heat-resistant magnesium alloy ML10 (Figure 3).

The geometric characteristics of the specimens (Figure 4), representing the fragments of cylindrical shell of $330 \times 200 \times 8 \mathrm{~mm}$ size and $20 \mathrm{~mm}$ longitudinal bending deflection allowed modeling the stressed state in the vicinity of the body repair welds.

On the specimens the welds were produced similar to those used in the body repair, as well as their EDT with the in-process control of values of residual stresses after welding and treatment.

On the outer surface of specimens the notches were applied having the length, width and depth of respectively 200, 2 and $4 \mathrm{~mm}$, simulating fractures of the body [3]. Over the notched area a two-pass weld was produced using manual TIG welding with Sc-containing filler metal at the mode, presented in Table 1. The 
Table 1. Mode of TIG welding of repair joints of intermediate body of ML10 alloy

\begin{tabular}{|c|c|c|c|c|}
\hline $\begin{array}{c}\text { Number } \\
\text { of passes }\end{array}$ & $U_{\mathrm{a}, \mathrm{V}}$ & $I_{\mathrm{w}}, \mathrm{A}$ & $V_{\mathrm{w}}, \mathrm{mm} / \mathrm{s}$ & $Q_{\mathrm{Ar}}, \mathrm{l} / \mathrm{s}$ \\
\hline 1 & 20 & 250 & 1.7 & 0.25 \\
\hline 2 & 20 & 350 & 1.7 & 0.35 \\
\hline
\end{tabular}

appearance of the specimen with a weld is presented in Figure 4.

The evaluation of stressed state of the outer surface of welded joint specimens was carried out using the contact-free method of electronic speckle-interferometry, based on the measurement of displacements during elastic unloading of metal volume in the investigated regions on the specimen surface caused by drilling blind holes [5]. The equipment complex was applied (Figure 5), in which the investigated area of the specimen 4 , fixed on the assembly plate 5 , was illuminated by the laser beam being a part of the optical interferometer 3 under the same angle from two directions and, thus, the components of the displacement vector in the plane were determined. The data of displacement measurements after welding and EDT were registered and processed using the specialized signal registration 1 and optical signal processing 2 units, being a part of the complex.

After welding and EDT the changes of the stressed state of welded joints were registered in the vicinity of the repair welds. According to the results of comparison of stress values before and after the treatment the efficiency of the EDT process was determined.

Using pulse generator «Iskra-4» the EDT of welded joints of specimens was carried out using the PEC series. At the same time, the amplitude values of pulsed current and dynamic pressure reached $3080 \mathrm{~A}$ and $20460 \mathrm{~N}$ respectively, and the phase shift between them amounted to $0.1 \mathrm{~ms}$, which provided synchronization of electric pulse and dynamic components of electrodynamic effect.

The sequence of EDT performance and layout scheme of the measured surface areas of the investigated specimens are presented in Figure 6.

Before EDT the initial stressed state of specimen along the lines $A-A$ and $B-B$ was registered. Along the axis $0-0$ at the surface area of the deposited weld metal of $100 \mathrm{~mm}$ length the EDT 1 was performed by the series of 20 PEC in the direction «from center to edges» (see Figure 6), after completion of which the change of the stressed state along the lines $A-A$ and $B-B$ was registered. Then EDT 2 of the base metal was performed at distance of $12 \mathrm{~mm}$ from the line $0-0$ by the series of 20 PEC in the direction «on the pass» with the subsequent control of residual stresses along the line $B-B$.

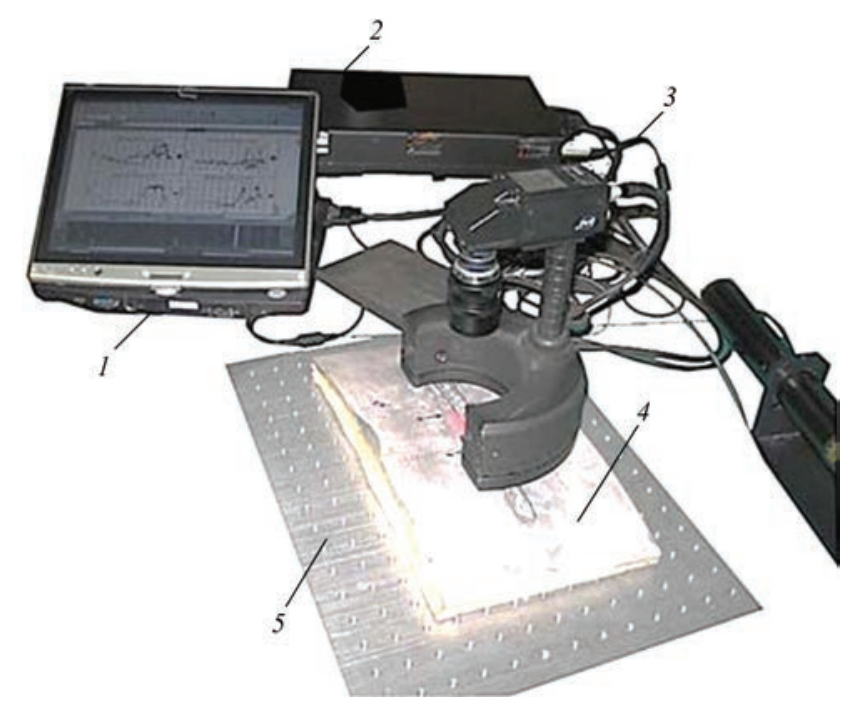

Figure 5. General appearance of equipment complex for registration of stressed state of ML10 specimens using the method of speckle-interferometry (for designations see the text)

The diagrams of the longitudinal component of plane stressed state $\sigma_{x}$ before treatment and after EDT 1 and 2 are shown in Figure 7.

Figure 7, $a$ shows distribution of $\sigma_{x}$ along the line $A-A$ (see Figure 6) after welding and EDT 1, from which it can be seen that before treatment a monotonous growth of tensile stresses $\sigma_{x}$ occurs along the weld line and their average values are $120 \mathrm{MPa}$. After EDT 1 the character of distribution of $\sigma_{x}$ along the line $A-A$ did not change significantly, but a decrease in their average values to $70 \mathrm{MPa}$ is observed.

The $\sigma_{x}$ distribution on the outer surface of the specimen after welding along the line $B-B$ perpendicular to the weld line (see Figure 6 ) is presented in Figure 7, $b$ (curve 1). The unbalance of the $\sigma_{x}$ diagram is connected with a significant bending component of the stresses on the surface of specimen caused by its initial curvature. The tensile $\sigma_{x}$ maximum value in the measured cross-section reaches the yield strength of alloy $\operatorname{ML} 10\left(\sigma_{0.2}=120 \mathrm{MPa}\right)$ at distance of $12 \mathrm{~mm}$ from the weld center.

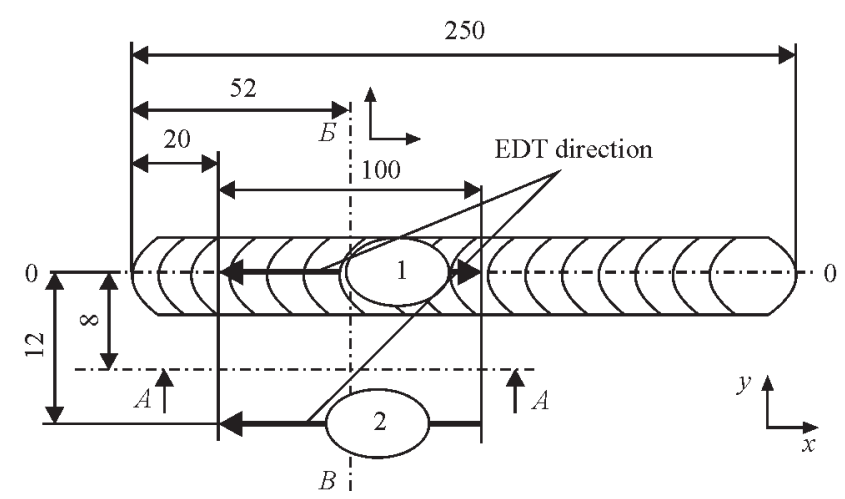

Figure 6. Sequence 1 and 2 of EDT and schemes of measured areas layout of surface of ML10 specimens along $A-A$ and $B-B$ lines 

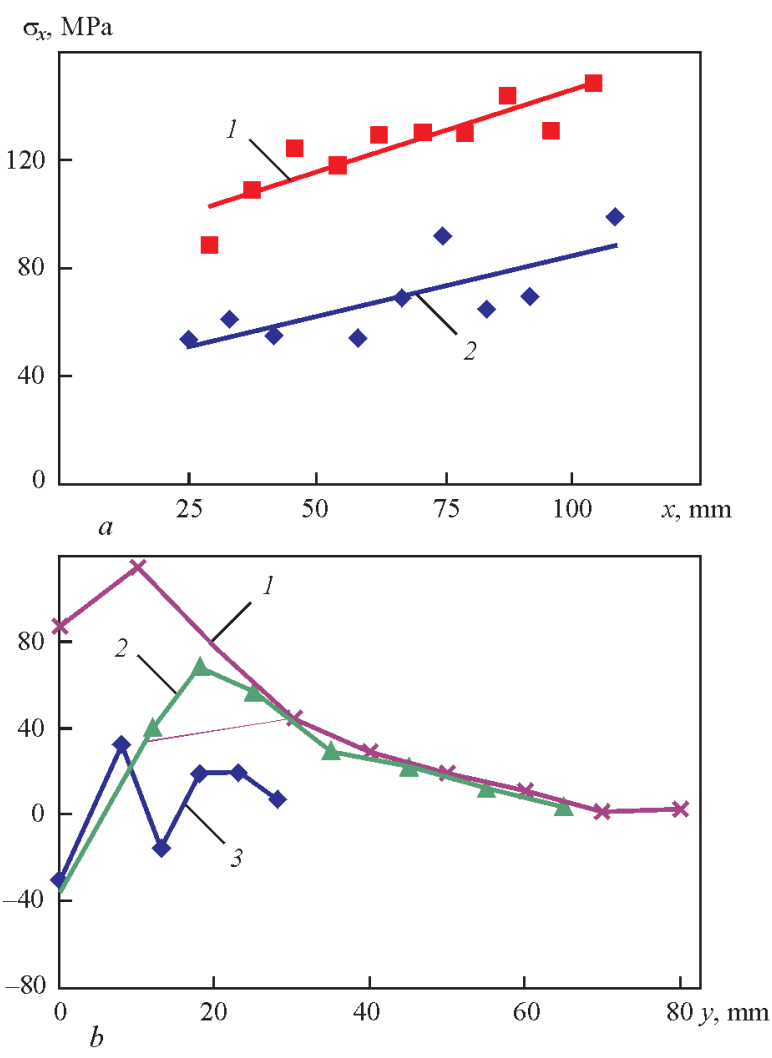

Figure 7. Stress $\sigma_{x}$ distribution in the ML10 specimen of welded joint: $a-\sigma_{x}$ before EDT (1) and after EDT 1 (2) along $A-A$ line; $b$ - the same before (1), after EDT 1 (2) and after EDT 1, 2 (3) along $B-B$ line

The influence of electrodynamic effects on the stressed state of the welded joint confirms the distribution of $\sigma_{x}$ after EDT 1 along the line $B-B$ (Figure 7, $b$; curve 2), from which it is seen that at the treated area of the weld center, $\sigma_{x}$ changed the sign from tensile to compressive stresses, whose values reached $-40 \mathrm{MPa}$. At the surface areas corresponding to the maximum values $\sigma_{x}=120 \mathrm{MPa}$, after EDT 1 stresses $\sigma_{x}$ decreased to $40 \mathrm{MPa}$, which makes $35 \%$ of their initial values. In general, it can be concluded that as a result of EDT in the treatment area a reduction of residual welding stresses and their transition from tensile to compressive ones is achieved (Figure 7, $b$; curves 1 and 2).

The $\sigma_{x}$ distribution along the line $B-B$ after EDT 2 is shown in Figure 7, $b$ (curve 3), from which it can

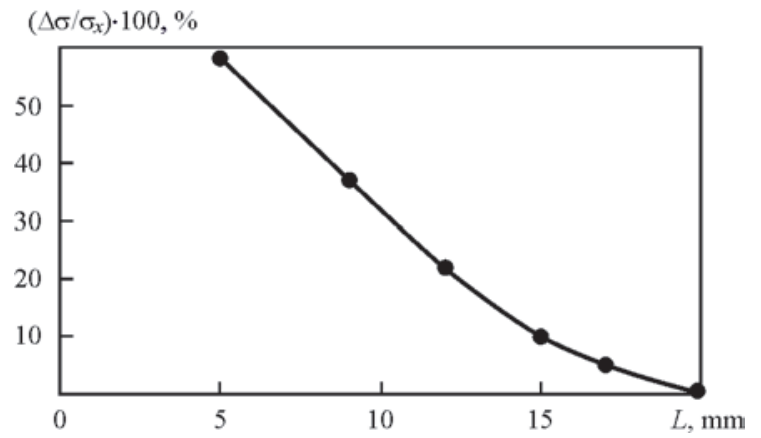

Figure 8. Effect of distance $L$ between EDT areas on efficiency of treatment $\Delta \sigma / \sigma_{x}$ be seen that after EDT 2 the formation of compressive stresses area occurs, localized at the area of electrodynamic effects, where $\sigma_{x}$ values are lowered to -20 MPa at the constant $\sigma_{x}$ values in the weld center equal to $-40 \mathrm{MPa}$. The lower efficiency of electrodynamic effect during EDT 2 of the base metal as compared to EDT 1 of weld metal can be explained by a higher plasticity of cast structure of the latter. Thus, the relative elongation of alloy ML10 $\delta=3.6 \%$, and the cast structure of the weld produced using filler containing scandium, reaches more than $6 \%$ [1]. At the same time, according to the data of work [6], the EDT efficiency increases with increase in ductility of the treated material.

The character of $\sigma_{x}$ distribution in the base metal after EDT 2 is close to that observed after EDT 1 in the weld centre. At the area of curve 3 between the areas of EDT 1 and 2, the reduction of $\sigma_{x}$ to $60 \mathrm{MPa}$ occurs, which makes only $50 \%$ of the $\sigma_{x}$ value after welding (before EDT 1 and 2), which confirms a localized character of electrodynamic effects.

Proceeding from the aforesaid, the determination of optimal distance $L$ between the areas of electrodynamic effects, providing a guaranteed overlap of the treated areas, is an important aspect in the development of EDT technology. In order to determine the optimal $L$ value at the specified electrical process parameters for the specimen of welded joint (see Figure 4) the EDT 1 of the weld metal was performed, changing the value $L$ between EDT areas. After the series of PEC the decrease in initial $\sigma_{x}$ was registered as a result of treatment $(\Delta \sigma)$ determining the EDT efficiency by the $\Delta \sigma / \sigma_{x}$ ratio.

The registration of $\Delta \sigma$ values was carried out along the weld line at the distance between the EDT areas equal to $L / 2$. The maximum value was preset to $L=20 \mathrm{~mm}$, successively reducing it to $5 \mathrm{~mm}$. The dependence $\Delta \sigma / \sigma_{x}=f(L)$ is presented in Figure 8, from which it can be seen that at $L \geq 20 \mathrm{~mm}$ the EDT efficiency is close to zero, indicating about the absence of overlapping of electrodynamic effects areas at the given EDT mode. At $L=15,12$ and $8 \mathrm{~mm}$ the $\Delta \sigma /$ $\sigma_{x}$ values reached 10,21 and $38 \%$ respectively, indicating about the partial overlap of EDT areas. At reduction to $L=5 \mathrm{~mm}$ and lower, the efficiency values were stabilized at $\Delta \sigma / \sigma_{x}=60 \%$, indicating that at distance not exceeding $5 \mathrm{~mm}$, there is a guaranteed overlapping of electrodynamic effects areas.

Thus, at the preset energy parameters of electrodynamic effects it is rational to carry out the EDT of ML10 alloy with a «step», not exceeding $5 \mathrm{~mm}$.

The presented investigation results showed that using EDT it is possible to regulate the level of residual stresses on the surface of welded joints of alloy ML10. 


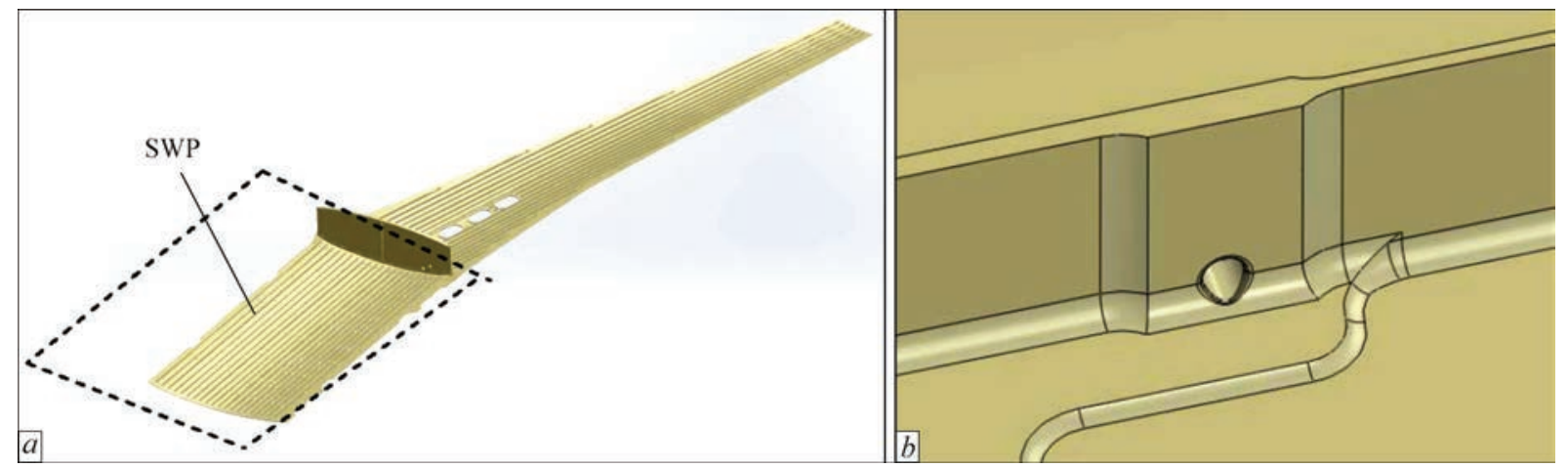

Figure 9. Appearance of bottom panel of aircraft wing: $a$ — general appearance of stringer wing panel; $b$ — hole for fuel overflow in stringer

After EDT the values of residual welding stresses are reduced significantly. The use of electrodynamic effects can positively affect the characteristics of fatigue strength of welded joints under the cyclic loads, which will provide the increasing in the service life of aircraft structures of magnesium alloys [2].

The competitiveness of aircraft products, especially passenger and transport planes, is largely determined by their optimal weight characteristics. Thus, the design weight at the specified takeoff weight and the design service life should be minimal.

The desire to achieve the life of $60,000-80,000$ flight hours under the condition of minimization of structural weight requires the application of new materials and different methods into industry to increase the fatigue life of both regular areas and the areas of structural irregularity.

To reduce the volume of aircraft fuel, which is not worked out and poured off, the holes for its overflowing are produced in the stringers of lower wing panels (Figure 9). The operation and aircraft ground tests show that such holes are the origins of fatigue cracks and may lead to premature fracture of the wing structure. This problem was encountered at the stage of service life tests of short main-line passenger aircraft SSJ-100 and multipurpose aircraft Be-200 and other [4].

To increase the endurance of aircraft wing panels in the holes area for fuel overflow the different technological methods are used, most of which are based on local deep plastic deformation (LDPD) in the hole area. These methods include barrier compression and boring used both separately and together, based on the local deformation of the material and creating residual compressive stresses in the area of increased concentration [7].

The barrier compression consists in indentation at some distance from the hole of the cylindrical punch of a specified profile, as a result of which in the holes the fields of compressive residual stresses are formed, increasing its durability (Figure 10).
The boring consists in pulling with tension through the hole of a specialized cylindrical tool-mandrel, resulting in squeezing out the part of material to the surface of the workpiece, intensive plastic deformation of the hole and, consequently, the formation of compressive stresses.

Despite the high efficiency of data of LDPD methods, increasing the fatigue life of the holes in the range from 1.5 to 10 times $[4,7]$ depending on the material and thickness of the stringer, they have certain disadvantages. For example, the equipment for realization of LDPD methods is sufficiently dimensional [4], and the technological cycle for treatment of one hole takes quite a long time.

It is challenging to use EDT for realization of LDPD, based on normal indentation of the profiled punch in the area of the hole during electrodynamic effects of PEC. The EDT advantages as compared to traditional LDPD methods are a high mobility of the positioning of the operating tool, which allows performing treatment of the holes in different spatial positions, including the operated aircraft structures. The period of treatment of one hole (excluding mounting of the tool) as compared to LDPD is many times shorter.

The different schemes of LDPD using EDT method (EDT LDPD) were investigated, aimed at creating

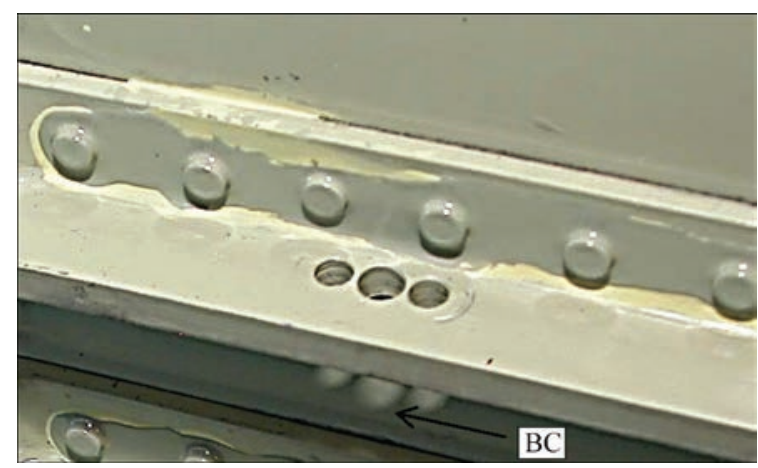

Figure 10. Stringer of the wing panel bottom part with three holes hardened using barrier compression, contour of which is marked with arrow 

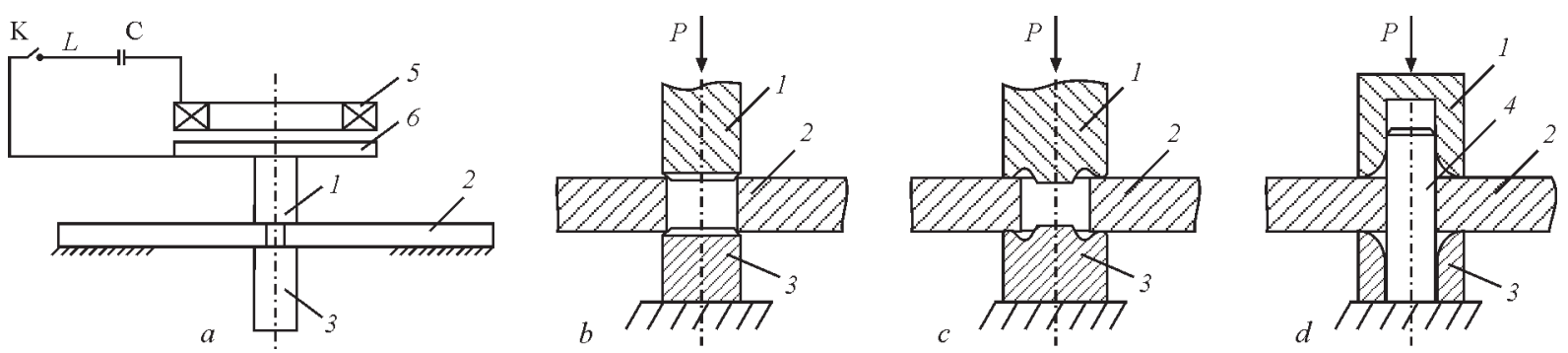

Figure 11. EDT of technological holes: $a$ - scheme of discharge circuit for EDT LDPD; $b$ - EDT-boring; $c$ - combined EDT-boring-compression; $d$ - EDT-compression; 1 - punch; 2 - stringer with hole; 3 - matrix; 4 - guiding bushing; 5 - inducer; 6 disc; K — contactor; C — capacitor; $P$ — electrodynamic effect

the area of compressive stresses in the hole area and the plastic deformation of its surface (Figure 11).

The scheme of discharge circuit for EDT LDPD is presented in Figure 11, $a$. The stringer supported against the rigid base was placed between the punch and the matrix. The punch is rigidly connected with the disc of a non-ferromagnetic material, which is the substrate for the plane inducer. During the start of discharge cycle of the capacitor with the contactor, the electrodynamic force is induced between the inducer and the disc tending to push them from each other, the vector of which is directed to the plane of the stringer along the normal. The effect of electrodynamic force on the punch and the matrix initiates their normal introduction into the treated material, at the same time providing LDPD in the hole area.

Considering the features of conventional schemes, described above $[4,7]$, the electrodynamic effect was used as a force factor providing dynamic introduction of the operating tool into the treated metal. Three schemes of EDT LDPD were proposed, such as EDT-boring (Figure 11, b), combined EDT-boring-compression (Figure 11, c) and EDT-compression (Figure 11, d).

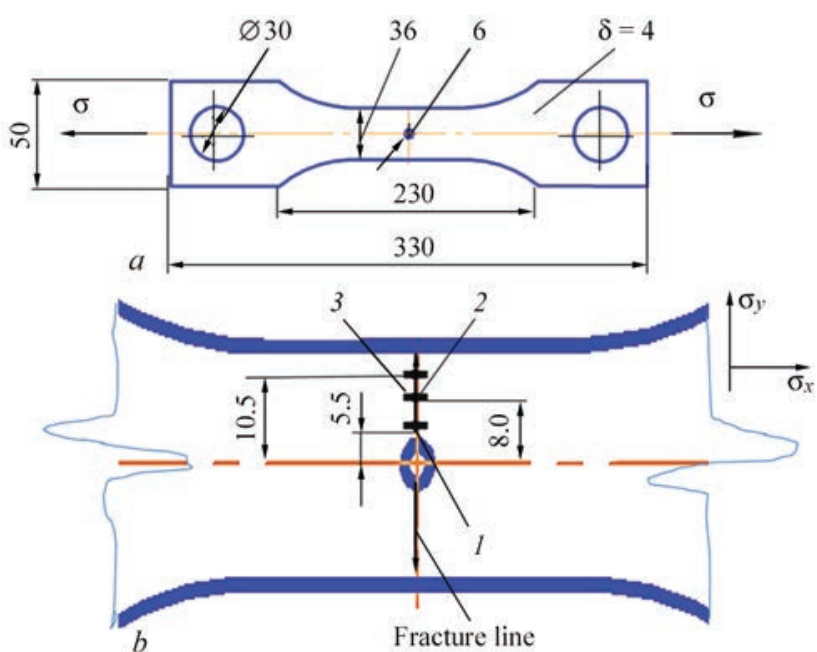

Figure 12. Specimen of alloy D16 stringer with technological hole of $6 \mathrm{~mm}$ diameter for test on fatigue resistance: $a-$ geometric characteristics; $b$ - testing areas of residual stresses in measured points $1-3$
The difference of EDT-boring from traditional EDT is the use of cylindrical punch and matrix, the working surfaces of which are in the form of the truncated cones instead of the mandrel extended through the hole. The electrodynamic effect of the tools on the treated metal provides LDPD of the hole surface of the stringer. The intensity of plastic deformation can be controlled by change in the level of stored energy and a number of PEC.

The combined EDT-boring-compression is the development of EDT-boring, where the profiled punch and the matrix except of LDPD of the hole itself provide the compression of the metal of the stringer surface behind the hole area. Moreover, the conical parts of the tool introduced into the treated surface in the LDPD process, provide alignment of the punch and matrix relatively to the hole edge.

During EDT-compression the alignment of the punch and the matrix with respect to the holes in the stringer is carried out by means of the cylindrical guiding bushing.

The investigations of efficiency of different schemes of EDT LDPD were carried out on the specimens of aluminum alloy D16 (Figure 12) used in the aircraft industry in the manufacture of stringer panels of aircraft wing.

The specimens were treated at three described schemes of EDT LDPD on the mode corresponding to the level of the stored energy of electrodynamic effect of not more than $800 \mathrm{~J}$ by the PEC series from 5 to 15 . The number of PEC in the cycle was determined by the type of the used tool. Thus, the minimum number of PEC, equal to 5, corresponded to EDT-boring (see Figure $11, b$ ), and that one equal to 15 - to EDT-boring-compression (see Figure 11, c).

After treatment of the hole area the level of residual compressive stresses $\sigma$ was registered in the area of the probable fatigue crack propagation (in points $1-3)$; in Figure 12, $b$ the direction of the $\sigma_{x}$ and $\sigma_{y}$ components is also shown. To evaluate the stressed state a non-destructive method of near-surface acoustic waves [8] was used, which allowed maintaining the integrity of specimens for the subsequent me- 
Table 2. Test results of specimens of D16 alloy with $6 \mathrm{~mm}$ hole after LDPD

\begin{tabular}{|c|c|c|c|c|c|}
\hline \multirow{2}{*}{ Scheme of EDT LDPD } & Number of specimen & $\sigma_{\text {max }}, \mathrm{MPa}$ & $N$ & $N_{\text {EDT }}$ & $N_{\text {EDT }} / N$ \\
\hline \multirow{2}{*}{ EDT-boring } & Free hole & 202 & 11000 & - & - \\
& 1 & 203 & - & 19000 & 1.72 \\
\cline { 2 - 6 } & $1^{*}$ & 202 & - & 16000 & 1.45 \\
\hline \multirow{2}{*}{ EDT-boring-compression } & $2^{*}$ & 203 & - & 17500 & 1.60 \\
\hline \multirow{2}{*}{ EDT-compression } & 3 & 201 & - & 27000 & 2.45 \\
\cline { 2 - 6 } & $3^{*}$ & 200 & - & 28000 & 2.54 \\
\hline
\end{tabular}

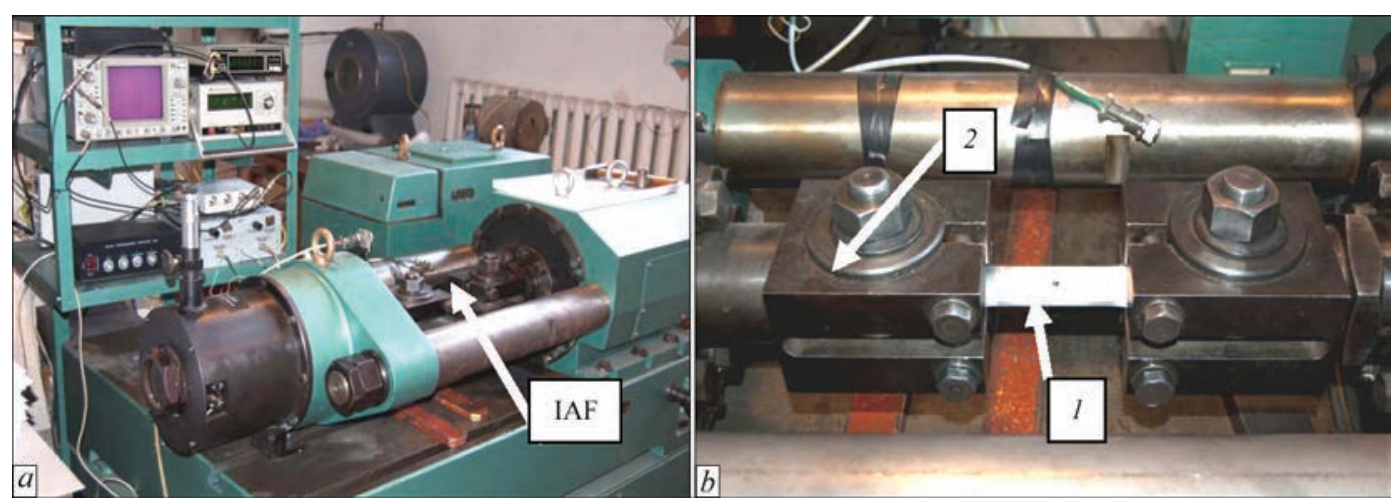

Figure 13. Installation for fatigue tests of specimens: $a$ - general view; $b$ - installation for applying force; 1 - specimen of stringer with hole; 2 - clamping unit

chanical tests on resistance to a delayed fracture. The maximum value of compressive $\sigma_{x}$ stress in point 1 reached -80 , in $2-40$ and in $3-105$, and $\sigma_{y}$ in points 1 and 2 was -95, in 3-120 MPa.

The tests of specimens were carried out at a gentle loading with the simultaneous registration of number of cycles $N$ before failure from zero cycle of loading by tension $\sigma_{\max }=200-203 \mathrm{MPa}$. The $N$ values were obtained for a free hole, and $N_{\mathrm{EDT}}$ - for treated ones. The tests, the results of which are shown in Table 2, were performed in the installation shown in Figure 13.

The dependence $\sigma_{\max }=f(N)$ in logarithmic coordinates for different schemes of EDT LDPD is presented in Figure 14.

Analyzing the data of Table 2 and Figure 14, it can be seen that EDT LDPD, realized on the basis of EDT-boring and EDT-boring-compression increase the resistance to delayed fracture of specimens in the hole area at $\sigma_{\max } \sim 200 \mathrm{MPa}$, determined by the $N_{\mathrm{EDT}} / N$ relation in 1.6 times, and EDT-compression - in 2.5 times.

On the basis of the presented data it can be concluded that the proposed schemes of EDT LDPD allow improving the resistance to fracture of technological hole, and taking into account possibilities of the method such as compactness and operating speed of the equipment, as well as the mobility of the operating tool positioning, they are promising for application in the production.

At the same time, there are opportunities to improve the efficiency of EDT LDPD, based on calculation-ex- perimental evaluations, for example [9]. Thus, the optimization of shape of working cones of the punch and the matrix for the proposed treatment schemes, taking into account the speed and the amount of dynamic loading at a normal introduction of the tool into the treated material, will provide the parameters of the stress-strain state of the metal in the hole area, necessary for the efficient fracture inhibition.

The results of the work were tested in the laboratory of aircrafts strength of the N.E. Zhukovsky National Aerospace University «KhAI».

On the basis of the carried out investigations on EDT of the repair welded joints of magnesium alloy ML10 and technological holes in aluminum alloy D16 it can be concluded that the electrodynamic effects allow regulating the stressed state of the thinsheet structural elements of light alloys in a wide

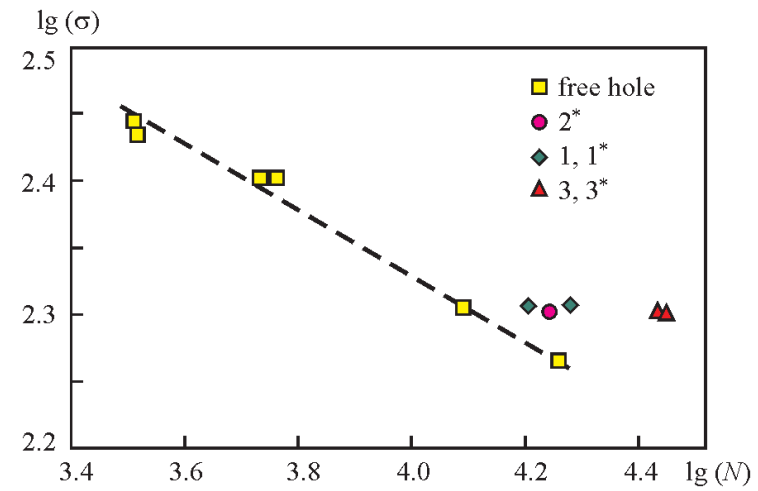

Figure 14. Tests results at from zero cycle of loading by $\sigma_{\text {max }}$ of D16 alloy specimens with $6 \mathrm{~mm}$ hole at different schemes of EDT LDPD acc. to Table 2: hatched line - resistance to fracture of hole without EDT LDPD 
range. Thus, EDT of welded joints of magnesium alloy provides transition of tensile residual stresses into the compressive ones, and the treatment of aluminum alloy initiates the field of compressive stresses in it, improving the resistance of metal against the delayed fracture under the cyclic loading.

The development of EDT method for its application in different technological processes will allow increasing the service life of metal structures of light alloys.

\section{Conclusions}

1. The pulsed electric current generator of the new generation with its controlled amplitude-frequency characteristics for electrodynamic treatment of thinsheet metal structures was designed and manufactured, which allows significant extension of capabilities of electrodynamic effects due to the controlled synchronization of the EDT dynamic and electric pulse components.

2. The effect of EDT on reducing of residual stresses in welded joints of magnesium alloy ML10 was investigated. It was established that using EDT it is possible to regulate the stressed state of ML10 welded joints, at the same time reaching compressive stresses.

3. It is shown that the local plastic deformation using EDT of specimens of aircraft wing stringer of aluminum alloy D16 in the area of technological holes 1.6-2.5 times increases the delayed fracture resistance.
1. Shalomeev, V.A., Tsivirko, E.I., Petrik, I.A. et al. (2009) Welding repair of surface defects in ML-10 alloy castings by using scandium-containing material. The Paton Welding J., 3, 23-27.

2. Lobanov, L.M., Pashchin, N.A., Cherkashin, A.V. et al. (2012) Repair welding of intermediate cases of aircraft engines from high-temperature magnesium alloy ML 10 with application of electrodynamic treatment. Ibid., 11, 28-33.

3. Lobanov, L.M., Pashchin, N.A., Savitsky, V.V. et al. (2014) Study of residual stresses in welded joints of high-temperature alloy ML10 with application of electrodynamic treatment. Problemy Prochnosti, 6, 33-41.

4. Zarutsky, A.V. (2014) Experimental study of service life of specimens with strengthened holes. Information 2: Software loading. In: Problems of design and manufacturing of aircraft structures: Transact. of KhAI, Issue 2(78), 57-65.

5. Lobanov, L.M., Pivtorak, V.A., Savitsky, V.V. (2006) Procedure for determination of residual stresses in welded joints and structural elements using electron speckle-interferometry. The Paton Welding J., 1, 24-29.

6. Lobanov, L.M., Pashchin, N.A., Loginov, V.P. et al. (2007) Change of the stress-strain state of welded joints of aluminium alloy AMg6 after electrodynamic treatment. Ibid., 6, 7-14.

7. Grebenikov, A.G., Krivov, G.A., Vasilevsky, E.T. (2009) Experimental study of influence of subsequent application of boring mandrelling and barrier compression on fatigue life of stringers in zone of fuel crossfeed hole. In: Open information and computer integrated technologies: Transact. of KhAI, Issue $43,54-64$.

8. Gushcha, O.I., Smilenko, V.N., Kot, V.N. (2009) Control of stresses on the base of application of subsurface acoustic waves. Tekhnich. Diagnostika i Nerazrush. Kontrol, 1, 11-13.

9. Fomichev, P.A., Zarutsky, A.V. (2014) Prediction of service life of structure elements with strengthened holes under regular loading. In: Open information and computer integrated technologies: Transact. of KhAI, Issue 64, 84-93. 